\title{
COMPLEX CARDIAC DEFECT IN A FETUS WITH TRISOMY 18: A CASE REPORT \\ Shagun Aggarwal
}

\begin{abstract}
This is a case report of a foetus which was brought for postmortem evaluation following antenatal detection of a complex cardiac defect. Presence of dysmorphism and other malformations like gut malrotation, bladder outlet obstruction, and esophageal stenosis led to suspicion of a syndromic diagnosis. Fetal karyotyping confirmed a diagnosis of Trisomy 18 (Edwards syndrome). This facilitated appropriate genetic counseling of the family and guidance for prenatal diagnosis in subsequent pregnancies.
\end{abstract}

Keywords:: Trisomy 18,Cardiac abnormality, autopsy.

\section{INTRODUCTION:}

Congenital cardiac defects are seen in 4-50 per 1000 live births and are one of the commonest malformations detected on antenatal ultrasound [1,2]. Majority of these malformations occur in isolation and are of multifactorial etiology. However, a large proportion, at least $30-40 \%$ antenatally and $5-10 \%$ postnatally occur as part of an underlying genetic syndrome [2,3]. Diagnosis of genetic syndromes is important for appropriate antenatal counseling regarding prognosis as well as recurrence risk prediction and prenatal diagnostic measures in subsequent gestations. This is a report of a fetus with Trisomy 18 and associated complex cardiac defect.

\section{CASE REPORT:}

Congenital cardiac defects are seen in $4-50$ per 1000 livebirths and are one of the commonest malformations detected on antenatal ultrasound [1,2]. Majority of these malformations occur in isolation and are of multifactorial etiology. However, a large proportion, at least $30-40 \%$ antenatally and $5-10 \%$ postnatally occur as part of an underlying genetic syndrome [2,3]. Diagnosis

Shagun Aggarwal 1,2

'Department of Medical Genetics, Nizam's Institute of Medical Sciences, Hyderabad

2:Diagnostics Division, Centre for DNA Fingerprinting and Diagnostics, Hyderabad

Corresponding Author: Shagun Aggarwal

Email: shagun.genetics@gmail.com of genetic syndromes is important for appropriate antenatal counselling regarding prognosis as well as recurrence risk prediction and prenatal diagnostic measures in subsequent gestations. This is a report of a foetus with Trisomy 18 and associated complex cardiac defect.

\section{CASE REPORT:}

A 26 week foetus was brought for post-mortem evaluation following ultrasound detection of Double outlet right ventricle and poly hydramnios. This was the second conception of a healthy non-consanguineous couple with previous healthy offspring. There was no history suggestive of teratogenic exposure and no significant family history.

External examination of the foetus revealed facial dysmorphism in the form of broad nasal bridge and tip, long philtrum, mild micrognathia and flat ear helix with groove on lobules bilaterally. The index fingers was overlapping $3^{\text {rd }}$ fingers and bilateral halluces were proximally placed. Female genitalia were unremarkable and there were no other external malformations. Umbilical cord showed presence of two vessels. Figure $1 \mathrm{a}$ and $\mathrm{b}$ depict the external appearance of the fetus.

Anthropometry revealed weight of $400 \mathrm{gms}$ (at $-2.5 \mathrm{SD}$ for 26 weeks), crown rump length of $21 \mathrm{~cm}$ ( $5^{\text {th }}$ centile for 26 weeks) and head circumference of $21 \mathrm{~cm}(-2.5 S D$ for 26 weeks). Internal examination of the abdominal cavity showed a tense and distended bladder. There was left hydroureter and right ureter was slightly prominent. Uretral openings in the bladder were normal. Urethra could be traced till the proximal half and no definite abnormality could be be visualized. The kidneys (Right $2.2 \times 1.5 \times 0.7 \mathrm{~cm}$ \& left $2.3 \times 1.2 \times 0.8 \mathrm{~cm}$ ) appeared normal with mild dilation of left renal pelvis (Figure 1d). Normal female internal genitalia were visualised. No cloacal abnormalities or genito-urinary fistulas were noticed. There was presence of large gut malrotation with the descending colon crossing over to right side and sigmoid colon being present on the right side. There was no obvious structural defect of the bowel (Figure 
1e). The lower end of the esophagus appeared stenosed and no definite connection with the lumen of the stomach could be appreciated. Liver and spleen were unremarkable.

Intrathoracic examination revealed two parallel great vessels originating from the heart. The cardiac size and axis appeared unremarkable. All four cardiac chambers were normal sized and with normal wall thickness. Normal atrio-ventricular connections were present and there were no septal defects. The aorta originated from the right ventricle and the main pulmonary artery from the left ventricle, indicating transposition. The ascending aorta and the neck vessels appeared unremarkable. However, there was a preductal coarctation of aorta (Figure 1c and f). The ductus arteriosus, branch pulmonary arteries and descending aorta were unremarkable. Bilateral lungs, thymus and diaphragm were normal looking. Esophagus could be traced upto the diaphragm but the communication with stomach could not be established. Trachea was normal and there was no trachea-esophageal fistula. Intra cranial dissection revealed a partly autolysed brain which was grossly normal.

Figure 1: 1a: Facial gestalt of the fetus showing broad nasal bridge and log philtrum, $1 \mathrm{~b}$ : Lateral view of the fetus showing the overlapping fingers, micrognathia, decreased folding of the helix, groove on ear lobule and prominent heels, 1c: View of the heart showing two parallele great vessels, $1 \mathrm{~d}$ : Distended bladder with bilateral kidneys and ureters, 1e: Right sided descending colon and sigmoid, 1f: Coarctataion of aorta.
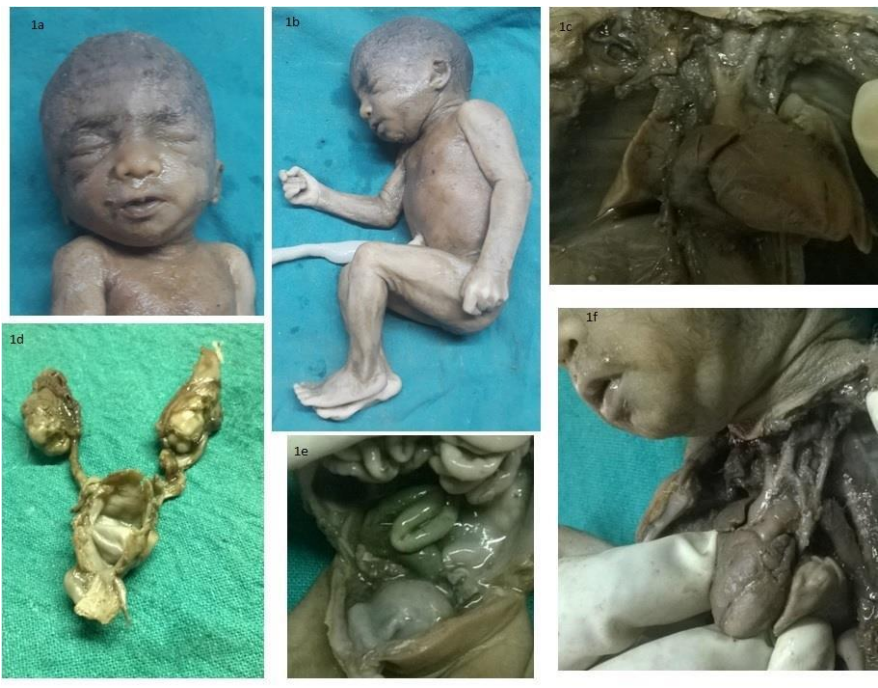

Fetal radiographs were unremarkable. Gross and histopathological evaluation by the pathologist was consistent with distal esophageal stricture and urethral stenosis. Renal architecture was unremarkable.

Karyotype of the fetus was done from cultured amniocytes obtained following an amniocentesis prior to pregnancy termination. This revealed a Trisomy of chromosome 18, confirming a diagnosis of Edwards syndrome (Figure 2). The couple were counselled about recurrence risk of up to $1 \%$ for subsequent pregnancies and were informed regarding availability of prenatal diagnosis services.

Figure 2: Fetal karyotype showing trisomy 18

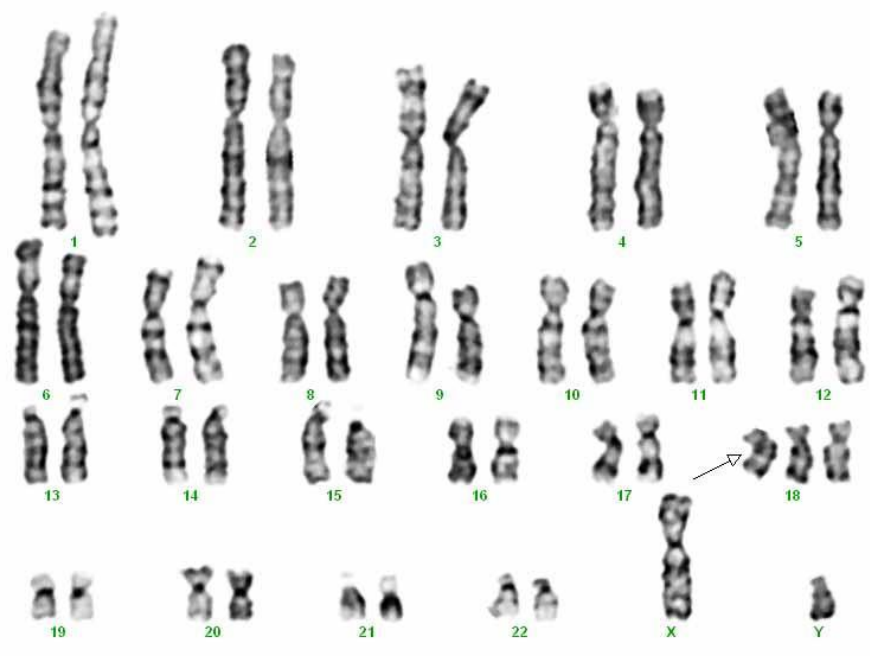

\section{DISCUSSION:}

Transposition of the Great arteries (TGA) is a common cardiac defect affecting 1 in 4000 liveborns. In one-third to one-eighth of cases it is associated with left ventricular outflow or arch abnormalities, as was seen in the present case. Most cases of TGA are multifactorial in origin, with a minor proportion being genetic in etiology [4]. Autosomal dominance with incomplete penetrance has been documented in few families with mutations in the MED13L and GDF1 genes, both being associated with non-syndromic or isolated TGA $[5,6]$. There are no specific syndromic associations, though TGA can be one type of cardiac defect in many chromosomal and nonchromosomal disorders. Some common syndromes with cardiac defects are the Trisomy 13, 18, 21 and the 22q11.2 deletion syndrome. In addition, the Heterotaxy 
syndromes can also have TGA as one of the component. These are genetically heterogeneous group of disorders, with different inheritance patterns and recurrence risks varying from $25-50 \%$ [7]. Coarctation of aorta (COA) is a left ventricular outflow tract abnormality and is primarily multifactorial in etiology, similar to other cardiac defects. However, it is the most common cardiac abnormality in patients with Turner syndrome (Monosomy X) [8]. It is also seen as an important component of the PHACES association, a neurocutaneous syndrome of unknown etiology, where additional features of posterior fossa abnormalities, haemangioma as, arterial abnormalities, other cardiac defects and eye abnormalities are present [9]. No monogenic forms of COA are described, though familial aggregation indicating oligogenic inheritance is reported in few cases. COA can also occur as part of various chromosomal and non-chromosomal syndromes and is not specific to any of these conditions except Monosomy $X$ [10]. Association of TGA with COA is uncommon but has been reported previously. Both these cardiac defects can be missed on routine antenatal sonogram if only the four chamber view is visualized. Hence, a detailed cardiac evaluation including imaging of the outflow tracts is recommended in high risk cases and should be attempted in all women if technically feasible to facilitate detection of these defects [11].

In the present case, the antenatal findings were suggestive of a double outlet right ventricle. However, postmortem evaluation delineated the precise cardiac defect and modified the antenatal cardiac diagnosis. A complex cardiac defect was found comprising of a TGA (Atrioventricular concordance and Ventriculoarterial discordance) and a COA. Additionally, autopsy identified multiple other abnormalities which remained undetected on antenatal sonography, leading to the suspicion of a syndromic etiology. This is in concordance with literature reports which indicate that post-mortem evaluation can provide additional findings and lead to a change in diagnosis in $25-50 \%$ cases of antenatally detected malformations [12]. Facial dysmorphism and gut malformations, as present in this case, are common abnormalities which are difficult to ascertain on antenatal imaging. In the present case, the syndromic association was further confirmed by fetal karyotyping which revealed Trisomy 18 (Edwards syndrome). Trisomy 18 is a chromosomal disorder associated with congenital cardiac defects $(>90 \%)$, growth restriction, joint contractures especially overlapping fingers, rocker bottom feet, radial ray defect, and many other internal malformations, which may differ from cases to case. The birth prevalence of Trisomy 18 is 1 in 6000-8000. Postnatal prognosis is extremely poor, with long term survival beyond 5 years age in only $5-10 \%$ individuals. The survivors have significant cognitive deficits and disabilities related to co-existing malformations. Antenatal screening for Trisomy 18 is possible by maternal serum screening in first or second trimester and a detailed fetal anomaly scan in the second trimester. A positive screening test mandates confirmatory testing by invasive fetal sampling followed by fetal karyotyping. Recurrence risk of free Trisomy 18 is $1 \%$ and prenatal diagnosis is recommended in all subsequent conceptions, as was advised to the parents in the present case [13].

Antenatally detected cardiac defects are associated with a chromosomal abnormality in $30-40 \%$ cases[3] and cono-truncal defects indicate a possibility of 22q11.2 deletion[2]. These genetic disorders can be associated with intellectual disability and other co-morbidities in the postnatal period. Hence, all women with antenatal detection of a cardiac defect should be counselled regarding possibility of a genetic syndrome and offered invasive prenatal testing followed by fetal karyotyping and testing for 22q11.2 microdeletion. This is an important adjunct to providing information regarding postnatal prognosis and surgical correction options for the cardiac defect, as it facilitates decision making regarding pregnancy termination or continuation; and appropriate prenatal management. In the event of pregnancy termination, the autopsy facilitates confirmation of diagnosis and counselling regarding recurrence risk in subsequent pregnancies [14,15].

To conclude, this is a case report of a fetus with apparently isolated cardiac defect on antenatal imaging, which was subsequently detected to have multiple malformations and a complex cardiac defect on fetal autopsy, and confirmed to be a case of Trisomy 18. The report highlights the significance of fetal autopsy and genetic testing in case of antenatally detected cardiac defects.

I would like to state that the findings in a case of trisomy 18 can vary from case to case. Few findings which are 
reported commonly are overlapping fingers (present in this foetus), cardiac defect (present in this foetus) and other internal malformations of various types (present in this foetus). Besides this, other findings are reported in literature, like prominent occiput, rocker bottom foot, micrognathia (mild in present case), radial ray defects, omphalocele, etc. However, these may or may not be present in a particular case. The karyotype findings confirm the diagnosis beyond any doubt in this case.

\section{REFERENCES:}

1. Hoffman JI. Congenital heart disease: incidence and inheritance. Pediatr Clin North Am. 1990; 37: 25-43.

2. Pierpont ME, Basson CT, Benson DW Jr, et al. Genetic basis for congenital heart defects: current knowledge: a scientific statement from the American Heart Association Congenital Cardiac Defects Committee, Council on Cardiovascular Disease in the Young: endorsed by the American Academy of Pediatrics. Circulation. 2007 Jun 12;115(23):3015-38.

3. Wimalasundera RC1, Gardiner HM. Congenital heart disease and aneuploidy. Prenat Diagn. 2004 Dec 30;24(13):1116-22.

4. Martins P, Castela E. Transposition of the great arteries. Orphanet J Rare Dis. 2008 Oct 13;3:27.

5. Karkera JD, Lee JS, Roessler E, et al. Loss-of-function mutations in growth differentiation factor-1 (GDF1) are associated with congenital heart defects in humans. Am J Hum Genet. 2007;81:987-94.

6. Muncke N, Jung C, Rudiger $\mathrm{H}$, et al. Missense mutations and gene interruption in PROSIT240, a novel TRAP240-like gene, in patients with congenital heart defect (transposition of the great arteries) Circulation. 2003;108:2843-50.

7. Unolt M, Putotto C, Silvestri LM, et al. Transposition of great arteries: new insights into the pathogenesis. Front Pediatr. 2013 Jun 6;1:11.

8. Sybert VP, McCauley E. Turner's syndrome. N Engl J Med 2004;351:1227-1238.

9. Roganović J, Adams D. PHACES syndrome--case report and literature review. Coll Antropol. 2009 Mar;33(1):311-4.

10. McBride KL, Pignatelli $R$, Lewin $M$, et al. Inheritance analysis of congenital left ventricular outflow tract obstruction malformations: Segregation, multiplex relative risk, and heritability. Am J Med Genet A. 2005 Apr 15;134A(2):180-6.

11. Salomon LJ, Alfirevic Z, Berghella V, et al. Practice guidelines for performance of the routine mid-trimester fetal ultrasound scan. Ultrasound Obstet Gynecol. 2011 Jan;37(1):116-26.

12. Rodriguez MA, Prats P, Rodríguez I, et al. Concordance between prenatal ultrasound and autopsy findings in a tertiary center. Prenat Diagn 2014 Aug;34(8):784-9.

13. Cereda A, Carey JC. The trisomy 18 syndrome. Orphanet Journal of Rare Diseases 2012, 7:81.

14. Fasnacht MS, Jaeggi ET. Fetal and genetic aspects of congenital heart disease. Ther Umsch. 2001 Feb;58(2):705 .

15. Driscoll DA. Prenatal diagnosis of the $22 \mathrm{q} 11.2$ deletion syndrome. Genet Med. 2001 Jan-Feb;3(1):14-8. 